\title{
BETA FUNCTION MEASUREMENT IN THE TEVATRON USING QUADRUPOLE GRADIENT MODULATION*
}

\begin{abstract}
A. Jansson, P. Lebrun, J. T. Volk,
Abstract

Early in Run2, there was an effort to compare the different emittance measurements in the Tevatron (flying wires and synchrotron light) and understand the origin of the observed differences. To measure the beta function at a few key locations near the instruments, air-core quadrupoles were installed. By modulating the gradient of these magnets and measuring the effect on the tune, the lattice parameters can be extracted. Initially, the results seem to disagree with with other methods. At the time, the lattice was strongly coupled due to a skew component in the main dipoles, caused by sagging of the cryostat. After a large fraction of the superconducting magnets were shimmed to remove a strong skew quadrupole component, the results now agree with the theoretical values to within $20 \%$.
\end{abstract}

\section{INTRODUCTION}

The Tevatron has two systems to measure emittance, the Flying Wires (FW), located at E11 and E17, and the Syncrotron Light Monitor (synclite) located at C11. At the start of run2, a rather large discrepancy was observed between the emittance values reported from these systems[1]. In order to understand the origin of this discrepancy, various studies were performed. On potential source of a scaling factor error would be an error in the beta functions. This can be measured quite directly by modulating the gradient of select quadrupoles.

It is well known[2] that if a quadrupole gradient is varied by a small amount $d K$, the local beta function can be extracted from the tune variation as

$$
\beta=4 \pi \frac{d Q}{d k}
$$

as long as the tune is far from the integer and half integer resonances. Close to these resonances, the more precise formula

$$
\beta=\frac{2}{d K}(\cot (2 \pi Q)(1-\cos (2 \pi d Q))+\sin (2 \pi d Q))
$$

should be used. These formulae only account for the direct change in tune due to the gradient change, and not any indirect change due to perturbations in the closed orbit. It is therefore important that the beam is centered in the quadrupole when the strenght is varied.

\section{AIR-CORE TRIM QUADRUPOLES}

Since individually powered quadrupoles were not available close to the locations of the three emittance mea-

\footnotetext{
* Work supported by the US Department of Energy
}

surement devices, it was decided to install such devices. Decommissioned air-core quadrupoles, originally built for resonant extraction of $800 \mathrm{GeV}$ beam for the Fermilab Fixed Target program, were modified for the purpose. The magnet coils were built around $76.2 \mathrm{~mm}$ ( 3 inch) diameter pipe. To allow for installation on a $101.6 \mathrm{~mm}$ (4 inch) diameter pipe with out breaking vacuum the coils were split along parting lines and re-potted on $101.6 \mathrm{~mm}$ forms. The coils were clamped around the existing Tevatron pipe and held in place with regular hose clamps. A rotating coil (see Fig. 1) was used to measure the excitation curve, the gradient and the higher order harmonics through the decapole. The excitation curve is shown in Figure 2. During the measurement phase the coils were totally removed and replaced on the test stand to check for reproducibility. There were no problems associated with removal and replacement of the coils around the pipe. All harmonics were less than 10 Fermilab units.

The quadrupoles were installed at $\mathrm{C} 0$, near the synclite detector, and at E0 and E17 near the flying wire locations.

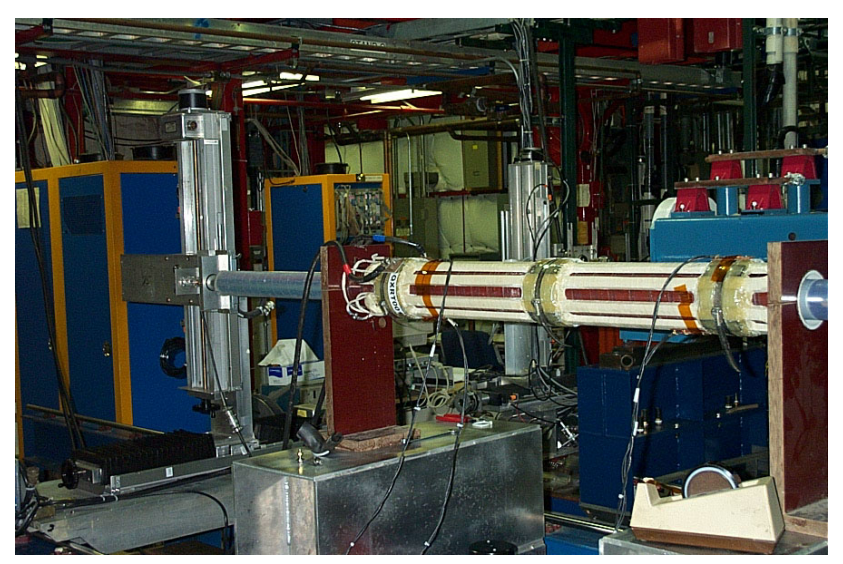

Figure 1: Modified air-core quadrupole on measurement stand in the Fermilab Magnet Test Facility.

\section{TUNE AQUISITION}

As the quadrupole current is modulated, the tune is measured with the $21.4 \mathrm{MHz}$ schottky tune tracker[3]. Although the magnets are capable to modulating the tune faster than 1 $\mathrm{Hz}$, the period had to be increased to cope with latency and refresh rate of the tune tracker software. Therefore, the system was set up with a modulation period of 1 minute, making the measurement of several periods relatively lengthy. 


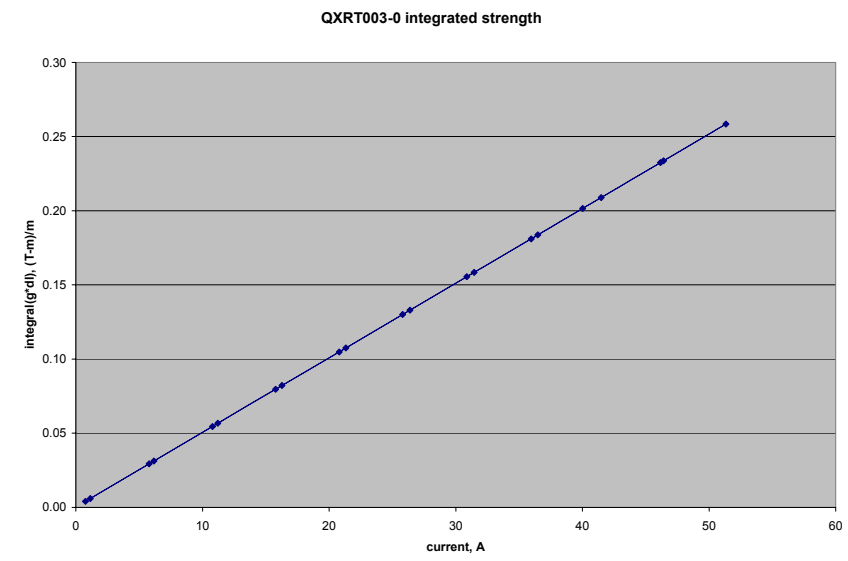

Figure 2: Measured integrated gradient versus current.

\section{MEASUREMENTS AT 150 GEV}

In 2003, the measured beta function (see Figure 3 and Table 1) disagreed significantly not only from the MAD lattice file, but also from models based on differential orbit measurements[4].

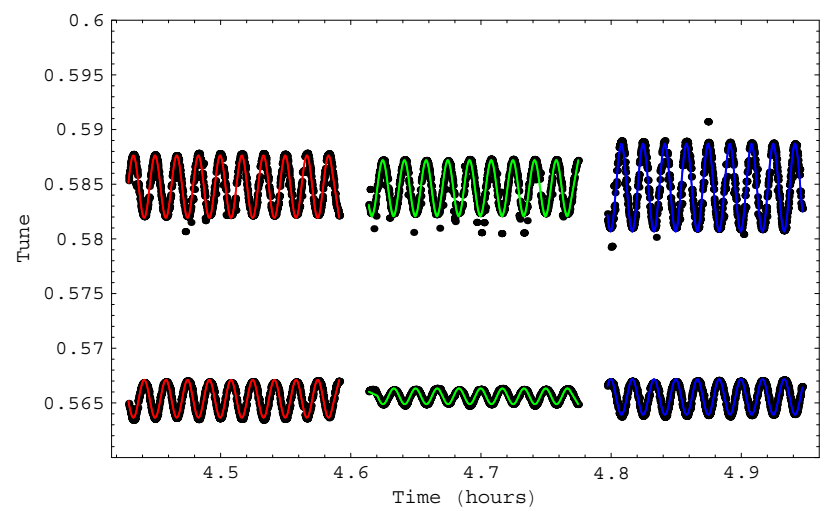

Figure 3: Measured tune variations on August 30, 2003. The E0 quadrupole was ramped 10 cycles first, then E17 and $\mathrm{C} 0$.

Table 1: Results from August 30, 2003.

\begin{tabular}{|l|c|c|c|}
\hline & E0 & E17 & C0 \\
\hline calc hor beta & $89 \mathrm{~m}$ & $77 \mathrm{~m}$ & $100 \mathrm{~m}$ \\
calc ver beta & $65 \mathrm{~m}$ & $32 \mathrm{~m}$ & $60 \mathrm{~m}$ \\
\hline meas hor ampl & 0.00284 & 0.00252 & 0.00395 \\
meas ver ampl & 0.00172 & 0.00072 & 0.00163 \\
meas hor beta & $71.1 \mathrm{~m}$ & $62.4 \mathrm{~m}$ & $98.6 \mathrm{~m}$ \\
meas ver beta & $43.0 \mathrm{~m}$ & $17.9 \mathrm{~m}$ & $40.6 \mathrm{~m}$ \\
hor diff & $-22 \%$ & $-21 \%$ & $-5 \%$ \\
ver diff & $-37 \%$ & $-47 \%$ & $-35 \%$ \\
\hline
\end{tabular}

In 2004, however, the results were found to agree to within 20\% (see Figure 4 and Table 2). Between 2003 and 2004, 108 dipoles out of 775 in the Tevatron were re-shimmed, which reduced the coupling of the machine drastically[5], so it is reasonable to assume that coupling was the cause. Indeed, looking closely at the 2003 data, there appears to be some signs of coupling as the tunes are moved together.

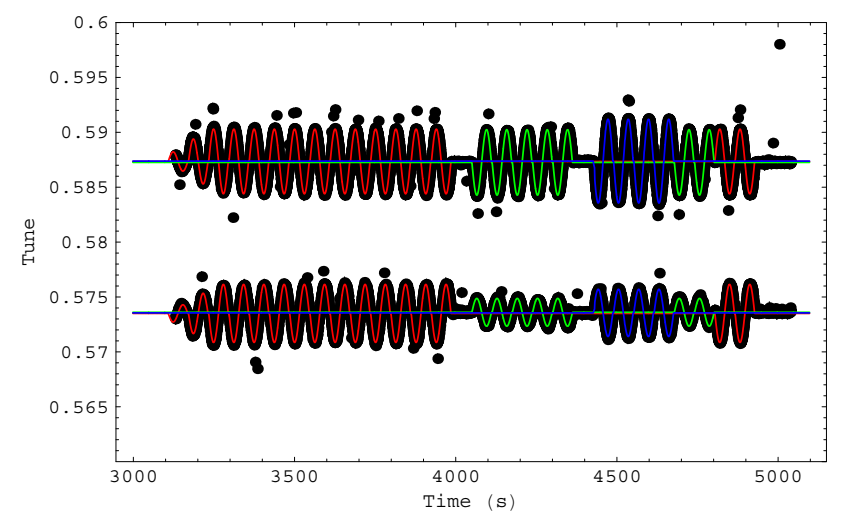

Figure 4: Measured tune variations on May 16, 2004. Red indicates E0 quadrupole ramping, green E17 and blue C0.

Table 2: Results at $150 \mathrm{GeV}$ from May 15, 2004.

\begin{tabular}{|l|c|c|c|}
\hline & E0 & E17 & C0 \\
\hline calc hor beta & $89 \mathrm{~m}$ & $77 \mathrm{~m}$ & $100 \mathrm{~m}$ \\
calc ver beta & $65 \mathrm{~m}$ & $32 \mathrm{~m}$ & $60 \mathrm{~m}$ \\
\hline meas hor ampl & 0.00294 & 0.00298 & 0.00383 \\
meas ver ampl & 0.00265 & 0.00126 & 0.00216 \\
meas hor beta & $73.3 \mathrm{~m}$ & $74.4 \mathrm{~m}$ & $95.4 \mathrm{~m}$ \\
meas ver beta & $66.1 \mathrm{~m}$ & $31.5 \mathrm{~m}$ & $53.8 \mathrm{~m}$ \\
hor diff & $-18 \%$ & $-4 \%$ & $-10 \%$ \\
ver diff & $1 \%$ & $0 \%$ & $-5 \%$ \\
\hline
\end{tabular}

In the presence of coupling, the observed tune split is different from the uncoupled lattice tune split

$$
\Delta Q_{\text {measured }}=\sqrt{\Delta Q_{\text {lattice }}^{2}+C^{2}}
$$

So that the observed tune shift is less than the expected, unless the coupling is much smaller than the tune separation. However, the scale of effect should have been less than a few percent in the measurements, since the global coupling was well corrected and the tunes separated by about four times the minimum tune split.

More likely, local coupling would be the cause, but the effect of local coupling is not as simple to estimate, as there are many parameters are involved. However, one can make the general observation that strong local coupling at the quadrupole location should also tend to reduce the observed tune modulation, since when the normal modes are inclined, a normal quadrupole appears like a skew quadrupole to the beam.

\section{BETA FUNCTIONS AT FLYING WIRES}

Due to space constraints, the trim quadrupole could not be located right next to the Synclite or Flying Wire instru- 
ments. However, if it is assumed that there are no gradient errors between the two quadrupoles in the E sector, the local amplitude and phase of the beta beat wave can be calculated (see Fig. 5).

The extracted beta functions at the location of the flying wires are given in Table 3, along with other measurements and the design values.
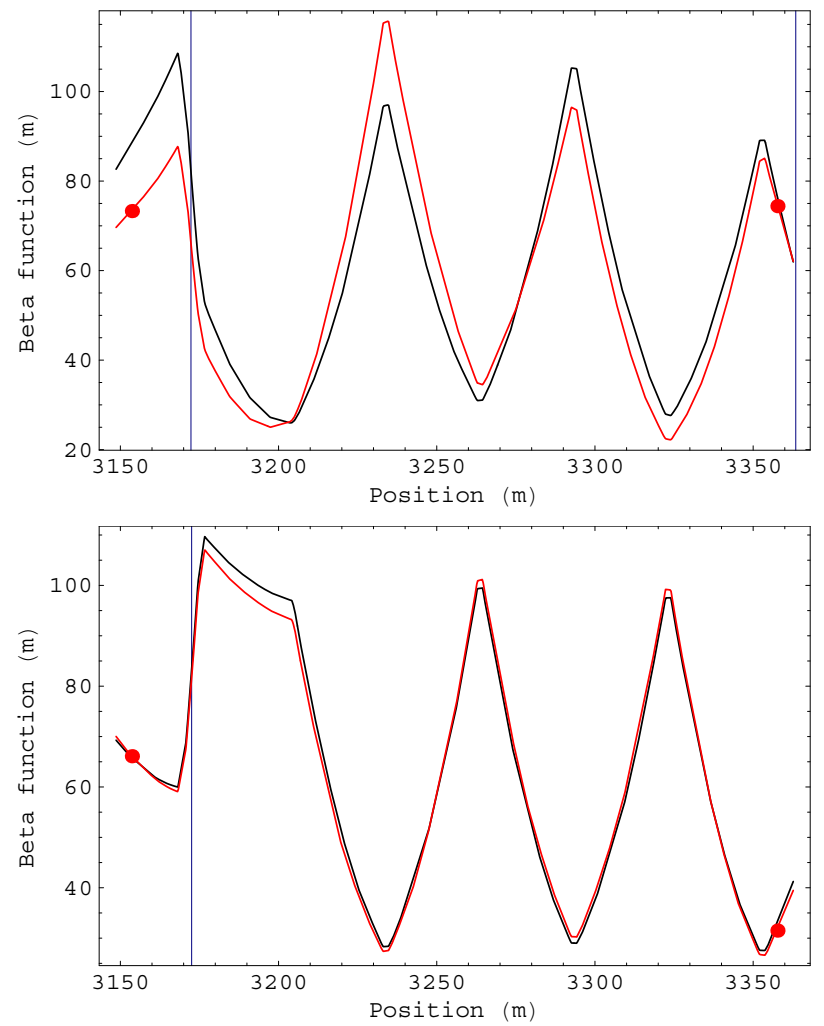

Figure 5: Fitted beta function in the e-sector. Top plot is horizontal, and bottom vertical plane. Red line is measured, black is design lattice. The dots are the data points, and the vertical lines indicate the location of the flying wires.

Table 3: Extracted $150 \mathrm{GeV}$ beta functions at the flying wires compared to theory and other measurements.

\begin{tabular}{|l|c|c|c|}
\hline & E11H & E11V & E17H \\
\hline This measurement & $66.0 \mathrm{~m}$ & $81.4 \mathrm{~m}$ & $60.2 \mathrm{~m}$ \\
MAD design optics & $82.1 \mathrm{~m}$ & $83.1 \mathrm{~m}$ & $59.8 \mathrm{~m}$ \\
Values used in FW & $83.5 \mathrm{~m}$ & $80.9 \mathrm{~m}$ & $62.5 \mathrm{~m}$ \\
Differential orbits 2004[4] & $86.9 \mathrm{~m}$ & $101.3 \mathrm{~m}$ & $67.6 \mathrm{~m}$ \\
\hline
\end{tabular}

\section{MEASUREMENTS AT 980 GEV}

Measurements were also performed at the Tevatron top energy of $980 \mathrm{GeV}$ (see Figure 6). Due to the higher magnetic rigidity, the measured tune modulation is 6.5 times smaller, and hence the noise on the tune measurement more important.

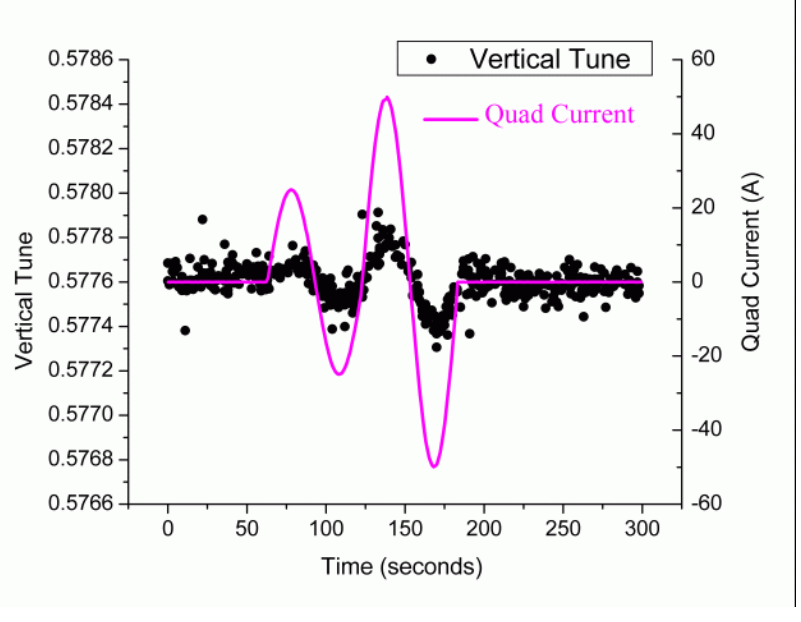

Figure 6: Gradient modulation test at $980 \mathrm{GeV}$.

\section{DISCUSSION}

Three air core quads were installed in the Tevatron to measure the local beta function in the vicinity of the emittance measurement devices. The intial results in 2003 were inconclusive, showing large dicrepancies relative to the theoretical lattice and other indirect measurements.

However, since 2004, the measured beta values are in resonable agreement with both the theoretical lattice and indirect measurements, on the level of 20\%. Between 2003 and $2004,14 \%$ of the Tevatron dipoles were reshimmed, reducing the coupling in the machine significantly (since then, another $53 \%$ have been reshimmed)

Although coupling is the likely supect for the disagreement in 2003, a quantitative explanation was not found.

At the end of 2004, one of the quadrupoles in the $\mathrm{E}$ sector had to be decommissioned to make room for the Tevatron IPM, another emittance measurement device. The remaining two are still operational. Eventually, the third quadrupole may be re-installed in a different location.

\section{REFERENCES}

[1] J. Slaughter et al, Tevatron Run II Luminosity, Emittance and Collision Point Size, Proceedings of IEEE Particle Accelerator Conference, Portland, OR, 2003.

[2] M. G. Minty and F. Zimmermann, Measurement and Control of Charged Particle Beams, Springer, Berlin, 2003.

[3] Paul Lebrun et al, "A Betatron Tune Fitting Package for the Tevatron 21.4 MHz Schottky" presented at this conference, and references therein.

[4] V. Lebedev, private communication and BeamDoc \#1465

[5] Michael J. Syphers and David J. Harding, Deterioration of the Skew Quadrupole Moment in Tevatron Dipoles Over Time, presented at this conference. 